\title{
Thermal-activation model for freezing and the elastic robustness of bulk metallic glasses
}

\author{
P. M. Derlet ${ }^{1, *}$ and R. Maaß ${ }^{2}$ \\ ${ }^{1}$ Condensed Matter Theory Group, Paul Scherrer Institut, CH-5232 Villigen PSI, Switzerland \\ ${ }^{2}$ California Institute of Technology, Division of Engineering and Applied Sciences, 1200 East California Boulevard, \\ Pasadena, California 91125-8100, USA
}

(Received 16 June 2011; revised manuscript received 29 July 2011; published 21 December 2011)

\begin{abstract}
Despite significant atomic-scale heterogeneity, bulk metallic glasses well below their glass transition temperature exhibit a surprisingly robust elastic regime and a sharp elastic-to-plastic transition. Here it is shown that, when the number of available structural transformations scales exponentially with system size, a simple thermal-activation model is able to describe these features, where yield corresponds to a change from a barrier energy dominated to a barrier entropy dominated regime of shear transformation activity, allowing the system to macroscopically exit its frozen state. A yield criterion is then developed, which describes well the existing experimental data and motivates future dedicated deformation experiments to validate the model.
\end{abstract}

DOI: 10.1103/PhysRevB.84.220201

PACS number(s): 62.20.D-, 81.05.Kf, 46.25.Cc

The mechanical properties of amorphous solids, such as bulk metallic glasses (BMGs) significantly below their glass transition temperature, are characterized by a robust elastic regime and a sharp elastic-to-plastic transition. ${ }^{1}$ Careful deformation experiments find that the stress at which yield occurs has a reproducibility comparable to a work hardened crystalline metal, ${ }^{2}$ suggesting an absence of macroscopic flaws and a BMG structure that is homogeneous above a certain length scale. At temperatures approaching the glass transition the situation changes considerably, where viscosity laws characterizing the undercooled liquid become increasingly operative, resulting in a less clearly defined boundary between elasticity and plasticity that is sensitive to how quickly the deformation experiment is performed (see Fig. 1).

The robustness of the elastic regime and the sharp transition to plasticity well below the glass transition temperature suggests a connection to a freezing transition, a phenomenon intimately linked to the presence of quenched disorder. In such systems, the energies of sufficiently large volume partitions are well described by a random variable, and at sufficiently low temperatures the free energy derived from averaging over such volumes is constructed from only a few low-energy configurations. Below a critical temperature one such configuration dominates, freezing the system into a zero-entropy state and giving a free-energy constant with respect to temperature.

Via the replica method, ${ }^{4}$ which is an analytic procedure to obtain the free energy through the moments of the subvolume partition function, the critical freezing temperature represents a transition from a replica symmetric regime, where different replica configurations contribute equally to the average, to a one-step replica symmetry-breaking regime, where one particular replica configuration dominates. The one-step replica symmetry-breaking method is equivalent to the Gumbel class of extreme value statistics, ${ }^{5}$ a connection which emphasizes that only the low-energy tail of the energy distribution is probed at low temperatures. Freezing also occurs when there exists (logarithmic) correlations between volume partitions, a feature resulting in a different universality class to that of Gumbel. ${ }^{6,7}$ Such universality classes of extreme-value statistics demonstrate that the freezing transition is a quite general phenomenon and thus applicable to a diverse range of systems, such as spin glasses via (say) the random energy model (REM), ${ }^{8}$ electron localization in disordered systems, ${ }^{9}$ and Burgers vector turbulence. ${ }^{10}$

In this Rapid Communication it is demonstrated that the robustness of the elastic regime and the sharp transition to plasticity for a BMG can be understood within the framework of equilibrium plasticity and can be related to a freezing transition. This is achieved by acknowledging that the available number of irreversible structural transitions mediating macroscopic plasticity scales exponentially with system size, and mapping the resulting barrier energy statistics to an equilibrium statistical mechanics description. It is found, however, that yield does not correspond to a transition away from a zero-entropy state, as in freezing, but rather to the point when the free barrier energy becomes negative, demonstrating that the transition from elasticity to plasticity may been seen as a transition from a barrier energy dominated regime to an entropy dominated regime. The paper concludes by developing an explicit yield criterion which describes existing experimental data and motivates dedicated deformation experiments to validate the theory.

Beginning with the seminal work of Spaepen ${ }^{11}$ (who used a free-volume argument) and Argon, ${ }^{12}$ the phenomenological concept of the shear transformation (ST) has emerged as the underlying microscopic mechanism facilitating equilibrium BMG plasticity, where a finite number of atoms structurally transform to relieve local stress. In both these papers, the theory of thermal activation plays a central role, in which the rate at which a particular ST occurs (labeled by $i$ ) is given by the product of an attempt rate $v_{i}$, and the probability that such an attempt is successful, $\exp \left(-\beta E_{i}\right)$, which is characterized by an energy barrier $E_{i}$ and temperature $\beta=1 / k_{\mathrm{B}} T$. Both $v_{i}$ and $E_{i}$ constitute two relevant microscopic parameters characterizing a particular ST, the third (not presently used) being the characteristic strain admitted by the ST. When an external stress is applied, the barrier energy will change in a way dictated by the geometry of the structural transformation, decreasing those that are compatible with the evolving deformation and increasing those that are not. The thermal-activation picture is motivated by the experimental observation that the plastic flow regime of BMGs is thermally activated, ${ }^{13,14}$ which necessarily 


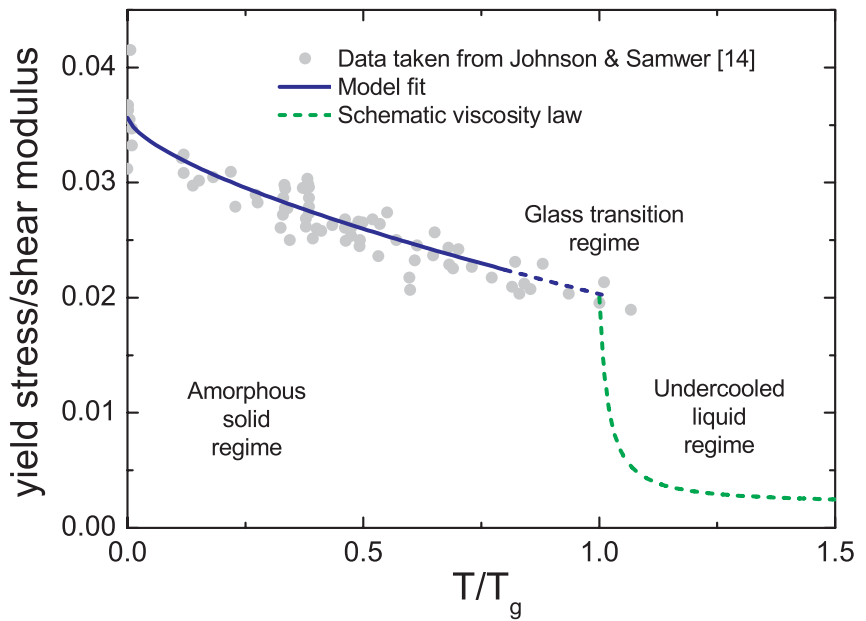

FIG. 1. (Color online) Yield stress vs temperature plotted as a yield stress over shear modulus vs $T / T_{g}$. Below $T_{g}$ the yield stress is given by the developed criterion, Eq. (7), and close to and above $T_{g}$ is described by an appropriate viscosity law (schematically indicated here). The dashed regions of the curves indicate a regime where the transition from elasticity to plasticity is smooth and very much dependent on strain rate. Experimental data is taken from Ref. 3.

infers that there is no deformation regime in which thermal activation may be avoided. ${ }^{1}$

If the system is in a particular local equilibrium state, the total yield rate $\dot{y}$ is given by a linear sum of the $M$ microscopic yield rates accessible to that state:

$$
\dot{y}=\sum_{i=1}^{M} v_{i} \exp \left(-\beta E_{i}\right) \approx v_{0} \sum_{i=1}^{M} \exp \left(-\beta E_{i}\right) .
$$

The common assumption of a single characteristic time scale for the attempt rates $1 / v_{0}$ should not be interpreted as each ST having the same attempt rate, but rather a result of $v_{i}$ being weakly correlated or even uncorrelated with $E_{i}$ due to the extreme sensitivity of $v_{i}$ to its corresponding structural transformation. ${ }^{15}$ A particular realization of $\left\{E_{i} ; i=1, \ldots, M\right\}$ can be sampled from a probability distribution $P(E)$ characterizing the possible barrier energies admitted by the BMG system. Evidence for such distributions can be found in recent potential energy landscape (PEL) explorations of small model glass systems, ${ }^{17}$ as well as experimental in situ relaxation kinetics measurements, ${ }^{18}$ both of which infer a broad distribution of ST barrier energies that peak at a finite energy value.

Theoretical insight into the form of $P(E)$ is to be gained by acknowledging that a wide range of structural transformations can occur, each characterized by a saddle-point configuration defining the corresponding energy barrier, and involving anywhere from a few to an extensive number of atoms. Formally this can be achieved via an analysis of the critical points of the underlying PEL, $E\left(\vec{R}_{1}, \vec{R}_{2}, \ldots\right)$, each of which is characterized by its energy and order: the number of negative eigenvalues of the corresponding Hessian. Doing just this, Shell et al. ${ }^{19}$ and Fyodorov ${ }^{20}$ have independently shown that the average number of such critical points depends exponentially on the number of atoms. Moreover, in the work of Ref. 19, the lower-order critical points are mainly constructed from an appropriate number of first-order critical points, the so-called equipartition of saddle-point order, with the result that cooperative activity is mainly realized by multiple first-order transitions.

To construct such a model ST distribution, it is recognized that there exists an upper length scale, characterized by a volume $V$, above which the system is extensive and individual volumes may be considered statistically independent. There also exists a lower length scale $V / N$, which is comparable to the atomic scale and characterizes the minimum size of a first-order structural transition, and has a characteristic barrier energy $E_{0}$. Via the equipartition of saddle-point order, an $n$ th-order saddle point is then given by the energy $n E_{0}$ and is referred to as a cooperative ST. The volume $V$ will therefore admit $\Lambda_{n}=N ! /(N-n) ! n !$ cooperative STs of order $n$ with the total number equaling $\sum_{n=1}^{N} \Lambda_{n}=2^{N}$, allowing for the construction of the normalized distribution:

$$
\begin{aligned}
P(E) & =\frac{1}{2^{N}} \sum_{n=1}^{N} \Lambda_{n} \delta\left(E-E_{0} n\right) \\
& \sim \sqrt{\frac{2}{N \pi}} \exp \left(-\frac{2 \Delta n^{2}}{N}\right) \delta\left(E-E_{0} n\right) .
\end{aligned}
$$

Here the latter Gaussian form has been achieved using Stirling's approximation for large factorials and $\Delta n=N / 2-$ $n$. It is noted that the material parameter $E_{0}$ underlies a simplification of atomic structure that has been exploited by Stillinger in the counting of inherent structures. ${ }^{21}$

The defining property of the above distributions is that both the first- and second-order moments are extensive quantities. It is a working hypothesis that this feature is robust against reasonable interactions between first-order critical points. Due to such interactions and depending on their spatial arrangement, a cooperative ST barrier energy $n E_{0}$ can also be indicative of first-order cooperative STs or a correlated succession of first-order events, in both cases allowing the system to exit its current state, transitions that are understood to be analogous to exiting a megabasin..$^{22}$ It is only such barriers that can contribute to Eq. (1), where in the former case standard transition state theory can be used to obtain the corresponding attempt rate and in the latter case the attempt rate will arise from mulitple correlated first-order activity. Writing the total number of such cooperative STs as $M_{N}=\left(1+\alpha_{0}\right)^{N}$, an estimate of the corresponding number density per unit energy is given by $M_{N}(E)=M_{N} P(E) . \alpha_{0}$ can be considered a material parameter which presently takes on a value between zero and unity. Since the equipartition of saddle-point order is only valid for low-order barrier energies, only the low-energy tail structure of $P(E)$ [and therefore $M_{N}(E)$ ] should be considered physically justified, a regime of barrier energies that turns out to play a crucial role in what follows.

Within this framework, a representative yield rate of the entire material system is obtained via a ensemble average of Eq. (1):

$$
\frac{\dot{y}}{v_{0}}=\left\langle\sum_{i=1}^{M_{N}} \exp \left(-\beta E_{i}\right)\right\rangle=M_{N} \int_{0}^{\infty} d E P(E) \exp (-\beta E),
$$


Using Eq. (2), the yield rate, Eq. (4), can be evaluated via the binomial theorem

$$
\frac{\dot{y}}{v_{0}}=M_{N}\left(\frac{1+\exp \left(-\beta E_{0}\right)}{2}\right)^{N}=\left(\frac{1+\alpha_{0}}{1+\alpha(T)}\right)^{N},
$$

where $\beta E_{0}=\ln \{[1+\alpha(T)] /[1-\alpha(T)]\}=f[\alpha(T)]$, giving $\alpha(T)=f^{-1}\left(\beta E_{0}\right)$ as a decreasing function of temperature. Equation (5) has the defining property that, for large $N$, the yield rate will be vanishingly small if $\alpha(T)>\alpha_{0}$ and exponentially large if $\alpha(T)<\alpha_{0}$. In this limit the transition point $\left[\alpha(T)=\alpha_{0}\right]$ corresponds to the equality $f\left(\alpha_{0}\right)$ $k_{\mathrm{B}} T=E_{0}$.

By explicitly indicating $E_{0}(\sigma)$ as a decreasing function of stress, a critical stress is defined for a given temperature $T$ via $f\left(\alpha_{0}\right) k_{\mathrm{B}} T=E_{0}\left(\sigma_{\mathrm{c}}\right)$, allowing the inequality in $\alpha(T)$ [now $\alpha(T, \sigma)]$ to be recast as

$$
\sigma<\sigma_{\mathrm{c}}(T)=\sigma_{\text {yield }}(T) .
$$

Equation (6) demonstrates that the yield rate can exhibit a fundamental change in behavior where, in the limit $N \rightarrow \infty$, it becomes a discontinuous function by being identically zero when Eq. (6) is satisfied and divergent otherwise.

By recognizing that the summation in Eq. (1) has the canonical form of a partition function, an extensive free barrier energy is defined via $\dot{y}=v_{0} \exp [-\beta F(T, \sigma)]$, where the elastic regime is defined by the condition $F(T, \sigma)>0$ and yield by $F\left(T, \sigma_{\text {yield }}\right)=0$ (see Fig. 2 ). Insight into the connection between the present work and the phenomenon of freezing is obtained by applying Derrida's microcanonical solution of the $\mathrm{REM}^{8}$ to determine $F(T)$ for a given stress $\sigma$. Beginning with the ansatz that the barrier energy density is given by the quenched average $\langle n(E)\rangle=M_{N} P(E)$, the entropy may be constructed via $S(E)=k_{\mathrm{B}} \ln \langle n(E)\rangle$ from which the critical

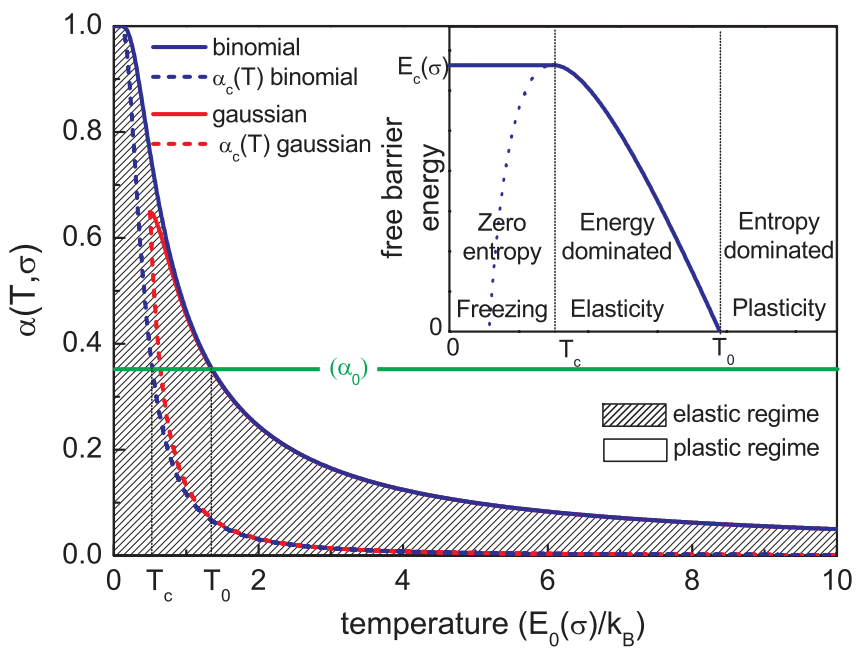

FIG. 2. (Color online) Plot of $\alpha(T, \sigma)$ vs temperature for the binomial and Gaussian ST distributions. For a given $\alpha_{0}$ (here chosen arbitrarily), $\alpha(T, \sigma)=\alpha_{0}$ defines the temperature $T_{0}$ at which there is a transition from elasticity to plasticity, and $\alpha_{\mathrm{c}}\left(T_{\mathrm{c}}, \sigma\right)=\alpha_{0}$ defines the critical temperature $T_{\mathrm{c}}$ below which ST barrier activity freezes into a single barrier energy. Inset: The free barrier energy constructed for a given $\sigma$ and $\alpha_{0}$, as a function of temperature, displaying the regimes of freezing and the barrier energy to entropy transition at yield. barrier energy is determined, $S\left(E_{\mathrm{c}}\right)=0 . E(T)$ is obtained via the "thermodynamic" relation $d S(E) / d E=1 / T$ giving the critical temperature $E\left(T_{\mathrm{c}}\right)=E_{\mathrm{c}}$ and $F(T)=E(T)-T S(T)$, resulting in three distinct temperature regimes: $T<T_{\mathrm{c}}$, where $F(T)=E_{\mathrm{c}}>0$ with cooperative ST activity being frozen into a zero-entropy state, $T_{\mathrm{c}}<T<T_{0}$, where $F(T)>0$, and $T>T_{0}$, where $F(T)<0$. See the inset of Fig. 2. It is emphasized that these quantities provide a convenient way to describe the resulting ensemble ST behavior, and do not carry the same physical meaning as their thermodynamic counterparts.

The Gaussian form of the cooperative ST distribution, Eq. (3), gives an exact result with the restriction $\ln \left(1+\alpha_{0}\right)<$ $1 / 2$ to ensure $E_{\mathrm{c}}>0-$ a consequence of the distribution incorrectly describing the tail structure $P(E \rightarrow 0)=0$ of the binomial distribution. An exact result can be also obtained for the binomial distribution, Eq. (2), for the full range of $\alpha_{0}$ using either the one-step replica symmetry-breaking method or the extreme-value statistics discussed by Bouchaud and Mézard. ${ }^{5}$ Analogous to Eq. (5) and its subsequent text, the temperature $T_{\mathrm{c}}$ is defined (now as a function of $\sigma$ ) via the condition, $\alpha_{\mathrm{c}}\left(T_{\mathrm{c}}, \sigma\right)=f_{\mathrm{c}}^{-1}\left[\beta E_{0}(\sigma)\right]=\alpha_{0}$. Figure 2 plots $\alpha_{\mathrm{c}}\left(T_{\mathrm{c}}, \sigma\right)=\alpha_{0}$ and $\alpha\left(T_{0}, \sigma\right)=\alpha_{0}$ as a function of temperature for both distributions, giving a pictorial representation of the above temperature regimes for a given value of $\alpha_{0}$, where an increase in stress [decrease in $E_{0}(\sigma)$ ] causes a contraction of the curves toward lower temperatures.

The elastic regime is therefore an internal barrier energy dominated regime. Although a freezing transition is present, the actual transition to plasticity occurs at a higher temperature when the entropy is large enough to give $F(T, \sigma)<0$, the point at which the availability of emergent ST activity scales more rapidly than the decrease in its probability of occurrencethe so-called entropy dominated regime. Thus, although the elastic-to-plastic transition does not formally correspond to a freezing transition, it does correspond to the moment when the system can macroscopically exit the frozen state.

The central simplification of the present model is to characterize local atomic structure via a characteristic firstorder structural transformation, an approach that has been used by Spaepen ${ }^{11}$ and Argon, ${ }^{12}$ in mesoscopic kinetic Monte Carlo simulations, ${ }^{23}$ and in the work of Johnson and Samwer. ${ }^{3}$ What is achieved in the present work is that such a fundamental energy barrier parameter, which is in this case $E_{0}$, reflects the scale of an extensive distribution of barriers rather than a single average energy barrier. Such an approximation relies on the observation that with increasing applied stress, it will be those structural transformations compatible with the evolving distortion that dominate the plasticity. It is nevertheless anticipated that a distribution of first-order STs will be admitted in a real BMG, as revealed by PEL explorations of model atomistic glasses. ${ }^{17}$ This will not affect the assumption of extremal, order $n$, cooperative ST activity being partitioned into $n$ first-order STs, and thus a cooperative ST distribution with extensive first and second moments - the fundamental ingredient for both the freezing transition and the discontinuous behavior of the yield rate as a function of stress and temperature.

Since $V$ represents a finite volume, set by the length scale at which the system is homogeneous, $N$ will also be finite and the transition becomes a crossover from elasticity to 
plasticity. Equation (6) remains accurate for $N>100-1000$, indicating a lack of sensitivity to reasonable values of $V$. Thus, when an increasing applied stress nears the critical stress $\sigma_{\mathrm{c}}(T)$, the yield rate rises rapidly indicating nonnegligible plastic flow-a regime of deformation in which accommodation mechanisms, ST-ST interaction, and inverse structural transformations play an important role that the present model is unable to describe. It is therefore emphasized that the current work is only applicable to the elastic regime, its domain of validity being set by Eq. (6), which defines the model's yield surface, as shown in Fig. 2.

Following the approach of Ref. 3 and assigning a universal barrier energy stress dependence derived from catastrophe theory $^{24}$ and supported by atomistic simulation ${ }^{25,26}\left[E_{0}(\sigma)=\right.$ $E_{0}(0)\left(1-\sigma / \sigma^{\prime}\right)^{3 / 2}$, where $\sigma^{\prime}$ is the stress at which the barrier energy vanishes], Eq. (6) can be transformed to an explicit yield stress criterion that is independent of $\alpha_{0}$ :

$$
\frac{\sigma_{\text {yield }}(T)}{\sigma_{\text {yield }}(0)}=1-\left(\frac{T}{T_{0}}\right)^{\frac{2}{3}} \text {. }
$$

Here $\sigma_{\text {yield }}(0)$ is the yield stress at zero temperature, and, by definition, $T=E_{0}(\sigma) / k_{\mathrm{B}} f\left(\alpha_{0}\right)$ and $T_{0}=E_{0}(0) / k_{\mathrm{B}} f\left(\alpha_{0}\right)$. In terms of the present model, $T_{0}$ is the yielding temperature at zero applied stress. When taking $T_{0}=\gamma T_{\mathrm{g}}$ (where $T_{\mathrm{g}}$ is the glass transition temperature and $\gamma$ a corresponding homologous parameter), Eq. (7) becomes operationally similar to that derived in Ref. 3, although its form is considerably simpler by virtue of it being a yield condition rather than an equation for equilibrium plastic flow. Figure 1 displays a good fit to the experimental data ${ }^{3}$ with the free parameters being $\sigma_{\text {yield }} / G$ and $\gamma$, where $G$ is a representative elastic shear modulus. In fact, Eq. (7) motivates a series of dedicated deformation experiments which measure yield as a function of temperature $T$ relative to a reference temperature $T^{\prime}$, to directly confirm Eq. (7) via $\sigma(T) / \sigma\left(T^{\prime}\right)=\left(T_{0}^{\zeta}-T^{\zeta}\right) /\left(T_{0}^{\zeta}-T^{\prime \zeta}\right)$, which has only one free parameter (when $\zeta=2 / 3$ ) and is free of elastic parameter rescaling. Such experiments, when performed at a given reference homologous temperature, will test sensitivity to compositional differences and thus the universal trend suggested in Fig. 1, and discussed in Ref. 3.

In summary, a model has been developed for BMGs which exploits the theory of thermal activation and an exponential scaling of the number of available microscopic structural transformations, resulting in a finite interval of temperature and stress in which a dominant extensive barrier energy causes negligible plasticity and thus a well-defined elastic regime. BMGs therefore exhibit the feature that, through their disorder, an extensivity emerges which considerably simplifies the structural dynamics leading to yield. What implications the present work will have on the plastic flow regime in terms of strain localization is not yet clear and constitutes an interesting direction of future work.

The authors acknowledge K. Clausen for institutional support, and C. Mudry for helpful discussion and critical reading of the manuscript. R.M. is grateful for financial support by the Alexander von Humboldt Foundation. *peter.derlet@psi.ch

${ }^{1}$ C. A. Schuh, T. C. Hufnagel, and Ramamurty U, Acta. Mater. 55, 4067 (2007).

${ }^{2}$ W. F. Wu, Y. Li, and C. A. Schuh, Philos. Mag. 88, 71 (2008).

${ }^{3}$ W. L. Johnson and K. Samwer, Phys. Rev. Lett. 95, 195501 (2005).

${ }^{4}$ M. Mézard, G. Parisi, and M. A. Virasoro, Spin Glass Theory and Beyond (World Scientific, Singapore, 1987).

${ }^{5}$ J.-P. Bouchaud and M. Mézard, J. Phys. A 30, 7997 (1997).

${ }^{6}$ D. Carpentier and P. Le Doussal, Phys. Rev. E 63, 026110 (2001).

${ }^{7}$ Y. V. Fyodorov and J.-P. Bouchaud, J. Phys. A 41, 372001 (2008).

${ }^{8}$ B. Derrida, Phys. Rev. Lett. 45, 79 (1980).

${ }^{9}$ C. C. Chamon, C. Mudry, and X. G. Wen, Phys. Rev. Lett. 77, 4194 (1996).

${ }^{10}$ Y. V. Fyodorov, P. Le Doussal, and A. Rosso, Europhys. Lett. 90, 60004 (2010).

${ }^{11}$ F. Spaepen, Acta Metall. 25, 407 (1977).

${ }^{12}$ A. Argon, Acta Metall. 27, 47 (1979).

${ }^{13}$ M. Heggen, F. Spaepen, and M. Feuerbacher, J. Appl. Phys. 97, 033506 (2005).

${ }^{14}$ R. Maaß, D. Klaumünzer, and J. F. Löffler, Acta Mater. 59, 3205 (2011).
${ }^{15}$ Within harmonic transition state theory, such sensitivity arises from the (Vineyard) prefactor being a ratio of products of a large number of positive eigenfrequencies [P. M. Derlet and D. Rodney (unpublished)]. For an example, see the prefactor discussion in Ref. 16

${ }^{16}$ B. P. Uberuaga, R. G. Hoagland, A. F. Voter, and S. M. Valone, Phys. Rev. Lett. 99, 135501 (2007).

${ }^{17}$ D. Rodney and C. A. Schuh, Phys. Rev. Lett. 102, 235503 (2009); Phys. Rev. B 80, 184203 (2009).

${ }^{18}$ S. V. Khonik, A. V. Granato, D. M. Joncich, A. Pompe, and V. A. Khonik, Phys. Rev. Lett. 100, 065501 (2008).

${ }^{19}$ M. S. Shell, P. G. Debenedetti, and A. Z. Panagiotopoulous, Phys. Rev. Lett. 92, 035506 (2004).

${ }^{20}$ Y. V. Fyodorov, Phys. Rev. Lett. 92, 240601 (2004).

${ }^{21}$ F. H. Stillinger, Phys. Rev. E 59, 48 (1999).

${ }^{22}$ P. G. Debenedetti and F. H. Stillinger, Nature (London) 410, 459 (2001).

${ }^{23}$ V. V. Bulatov and A. S. Argon, Model. Simul. Mater. Sci. Eng. 2, 167 (1994).

${ }^{24}$ V. Arnold, Catastrophe Theory (Springer, Berlin, 1992).

${ }^{25}$ D. J. Wales, Science 293, 2067 (2001).

${ }^{26}$ C. E. Maloney and D. J. Lacks, Phys. Rev. E 73, 061106 (2006). 